\title{
Intelligent Setting Package Based on Setting Value Graining and Common Library
}

\author{
Ping ZHAO ${ }^{1,}$, Linling DING ${ }^{1, b}$, Xuedond LI ${ }^{2, c}$, Kang Wang ${ }^{1, d}$,Wei Meng ${ }^{2, \mathrm{e}}$ \\ 1State Grid of Taizhou Power Supply Company, Zhejiang, China \\ 2 Beijing Join Bright Digital Power Technology Company, Beijing, China \\ a864911198@qq.com,,b359712785@qq.com,'snowinterli@126.com, \\ d78384782@qq.com, emwjy123@126.com
}

\begin{abstract}
Keywords: wind speed forecasting; six-unified; setting calculation; device setting; protection device common libraries; setting value graining

Abstract. In-depth analysis and study of six unified device fixed value and setting principle, combined with the application of the device setting in the relay protection setting calculation software. Intelligent setting package with protection device common libraries and granular fixed value was designed and developed based on database technology in relay protection setting calculation software. The common setting value of line, transformer and bus protection was summarized in protection device, and the value of reference was support in different protection device. Protection device model was split to header, fixed value area and footer with setting value graining. Then the repeated established work of header, fixed value area and footer in different areas was avoided. Finally, this paper design and development of intelligent setting package is applied to the field setting value calculation, the results show the practicability, flexibility and scalability of the function of this article.
\end{abstract}

\section{Introduction}

In order to ensure that the power grid which has flexible operation mode and expand continuously can be operated safely and reliably, the sensitivity, selectivity, quick acting and reliability of the fixed value for the relay protection equipment which has been considered as the last defense of stable operation of power grid are becoming more and more important. Thus, the relay protection setting calculation software ${ }^{[1-5]}$ has been arisen for the accurate calculation of setting value.

The existing setting calculation software is skillful in fault calculation ${ }^{[6]}$, principle setting of the transmission line and transformer, device setting ${ }^{[7]}$ technology and can greatly improve the accuracy of the setting value since the bad situation such as various inspection and the combination between plant and station has also been taking into account during the calculation. For practice application, the final purpose of setting value is to generate protection setting value list. And the existing setting calculation software can connect the model of protection device with setting value and obtain the fixed value list automatically. However, the maintenance work of protection device model is very complicated and time-consuming leading to the impracticability of the existing setting calculation software.

The popularization and application of software in the device setting function is limited due to the massive work during the process of modeling of protection device attributing to the massive manufacturers, type and fixed value item of relay protection device. The existing setting calculation software has considered common constant value including protection device manufacturers, protected equipment type, fixed value, rules of setting value and the maintenance of setting value formula while creating the model of setting value list. However, lots of redundant setting items generate in the gather of common constant item due to various labels, rules, and formula of protection device with has different manufacturer and types.

In order to standardizing the relay protection device and fixed value, six unified standard including functional configurations unified standard, loop design unified standard, terminal board layout unified standard, interface standard unified standard, clamp cabinets unified standard, protection fixed item and report form unified standard has been issued in 2007 and 2008 by state grid corporation of china based on four unified standard. In this work, we design common feature bank of 
protection device and intelligent setting toolkit of granule fixed list. The new construction, modification, storage and reuse of protection device fixed item are realized by the bank based on the six unified standard. The bank supports the citation of fixed item by different protection device. The construction of protection device common feature bank reduces the workload and realizes the statistic analytical function of protection device type, protection equipment fixed item information for common feature item. Protection fixed value list model is split into header, fixed value zone and footer by the granule fixed list. The granule fixed list avoids re-structure of fixed value item model caused by requirement of format of header, footer in different zone and especially is suitable for six unified protection device. Practicability and extensibility of this function is proved by the practical application in Taizhou power grid.

\section{Setting Value Common Libraries of Protection Device}

Analysis of Protection Device Setting Value Item. As the application of microcomputer protection being used widely, because of the numerous number of the microcomputer protection device models from different factories and numerous number of fixed value items from different devices, although huge differences of different fixed value items, principles of value and formulas, there are something in common. After analyze the fixed value items of different models of protection devices from different manufacturers, we found that the fixed value items of different device models from the same protection device class of the same manufacturers are the same (same name of fixed value item, same principle and formula); The values of variables of same device class formula from different manufacturers are the same; those values all come from the fault calculation results. According to the characteristics mentioned above, the current setting calculation software separates the fixed value items of the protection devices to different fixed value items and common fixed value items, which include fixed value item and control word with same setting calculations. Different fixed value items include specific fixed value item of the device, that specific fixed value item only takes the recommend default value of the user, and put the common value rule and variables into a set to make it easy to use when creating the formula.

As the user applying, some problems caused by non-standardized de-normalization fixed value items gradually appears in the setting calculation software, those problems lead to a lot of repeat fixed value item in the common fixed value item of the protection devices, showing in table 1 and table 2.

Table 1 Line protection setting values in $b$ manufacturer

\begin{tabular}{cccc} 
Setting designation & Current variation starting values & Zero sequence starting current & Ground-distance relaying I \\
\hline Rules & $\begin{array}{c}\text { According to escape the } \\
\text { maximum value of normal load } \\
\text { current fluctuations to set } \\
\text { Formula }\end{array}$ & $\begin{array}{c}\text { According to escape the maximum } \\
\text { unbalance current in zero sequence to set }\end{array}$ & $\begin{array}{c}\text { Get principle protection } \\
\text { setting }\end{array}$ \\
\hline Kk & $\mathrm{Kk}^{*}$ Ifmax & $\mathrm{Z}$ \\
\hline
\end{tabular}

By comparing three setting value of the line in $a, b$ manufacturer familiar, can arrive the setting value list with different designation, same rules and formula was exist in different manufacturer, and the maintenance of these repeated setting value is the main reason for increase time in establishing device model.

The Implementation of Device Common Repository. According to the six unified standard, the protection device common libraries was designed and developed in relay protection setting software based on database technology and c \# programming language. The common setting item in different protection device was store in common libraries, the structure of common setting value was shown in figure 1. Common setting item was stored automatically in the library when it was set up. While the rest of the protection device for application device fixed value item, only need to reference the common fixed value items in common library, do not need to rebuild, and the workload of building device model was greatly reduced.

On the other hand, protection device common libraries implement the reuse and synchronous update of common setting value. All of the protection devices were automatic synchronization update with using the item after the change of common setting value items. The common setting value items and 
protection device list, element equipment that reference items were to compile statistics, statistical process was shown as figure 2 and figure 3.

$\square$ Protection device type

$\square$ Setting value item description and attribute set

$\square$ Principle description and attribute set

$\square$ Computational formula

Conventional variable

Fig. 1 Structure of universal setting values

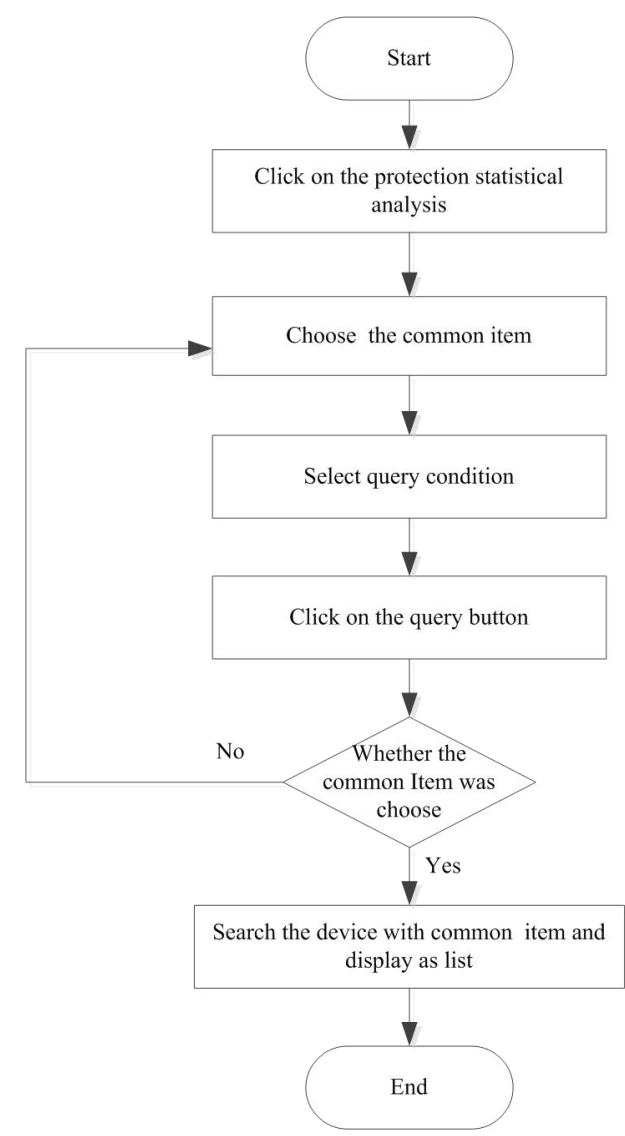

Fig. 2 Application and statistics on common setting values in protection device common library

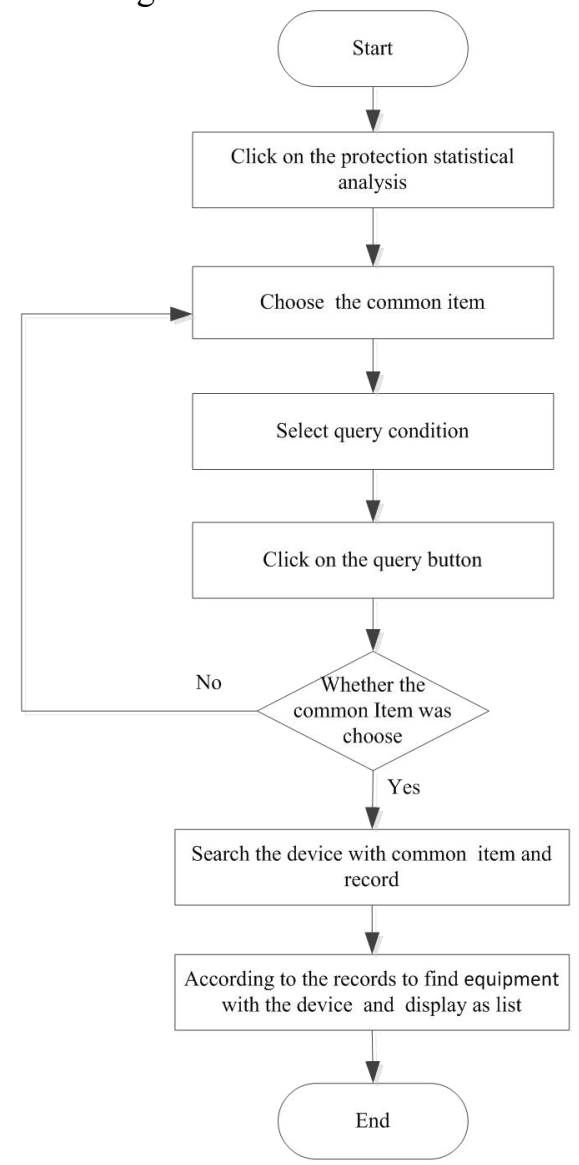

Fig. 3 Statistics on protection device

With the actual needs, 285 setting value items were encapsulated in the system according to the device type, mainly includes:

(1) The common item of Line protection;

(2) The common item of transformer protection;

(3) The common item of bus protection;

(4) The common item of circuit breaker protection, etc.

The common item of line protection includes parameter item (name, length, impedance, zero sequence compensation factor, CT and PT, etc.), principle setting value item (grounding distance protection, phase spacing protection and zero sequence over current protection, etc.), device setting value item(variation starting current, zero sequence starting current, different protection, etc.), as well as the control word in common, the application effect is shown in figure 4. 


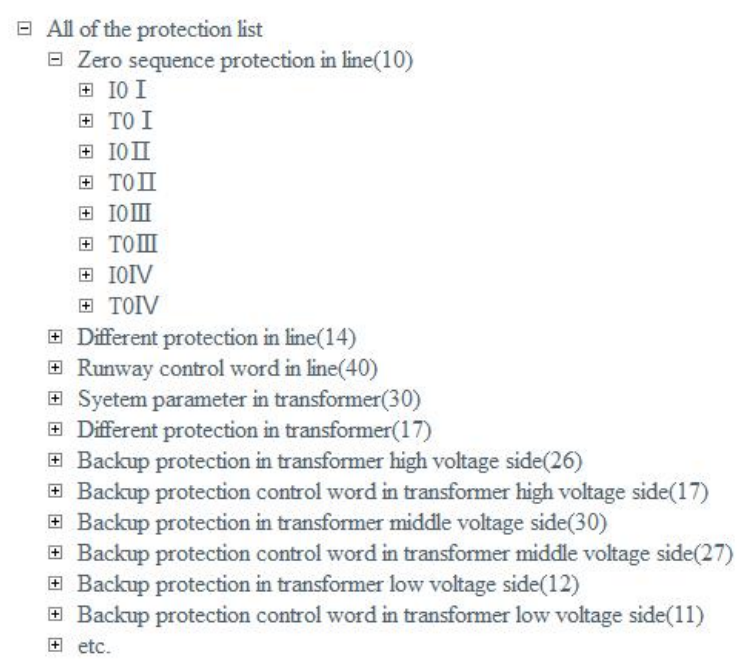

Fig. 4 Statistics exhibition on system common setting values

With the function of device common item, when setting value template was created, only need to choose an item in setting value item concentration according actual requirement. If it was not exist item which needed or formula was not correspondent with practice, then, the value principle and formula of setting value item were added or modified according to the device specifications.

On the base of the above analysis, it can be seen the advantages of device setting common library:

(1) The reuse of setting value. A series of reusable setting value was defined in system, then the create time of setting value template was reduced, and the efficiency of setting calculation was improved.

(2) The synchronous update feature of setting value. The protection device which referenced common setting value item can realize modify automatically when the content of item was changed, and the modified work with manually was avoid, then a lot of time was saved.

(3) Strong practicability, expansibility and maintainability. Protection device setting value item was added, modified according to the actual need, also special setting value item of the local device could add as needed.

\section{Graining Setting Value}

Setting value list is the standard for field operation personnel to enter setting value in protective device, and as the same as setting value list, different types of protection device has different templates, all of the setting values were collected in templates. The workload of relay protection workers was relieved with the arising of templates in setting software, and the single intelligent management was strengthened ${ }^{[12]}$.

The reason of setting list template in setting software was varied with user's region is different users has different setting list template, some limitations on the development of the expansible function was increased for software, and because of setting list template generally make up the header, setting value area, and footer, also envelope was exist in some places template. In order to increase the flexibility of the setting list template, graining was used in this paper, it resolves template to the header, setting value area, and footer, among the three parts, the header part contains header information, basic information and the text information maintained by existing setting list, and different device types has different header information, for example, some of number, date, substation name, equipment name, switches number, protection type, device model, invalid number, company name, CT, PT, sequence impedance and zero sequence was formed the header information of line; the calculator, checker, verifier, approver was typically formed footer contains, setting value area association was established using the universal library protection template, the creation and operation of setting list template as shown in figure 5: 


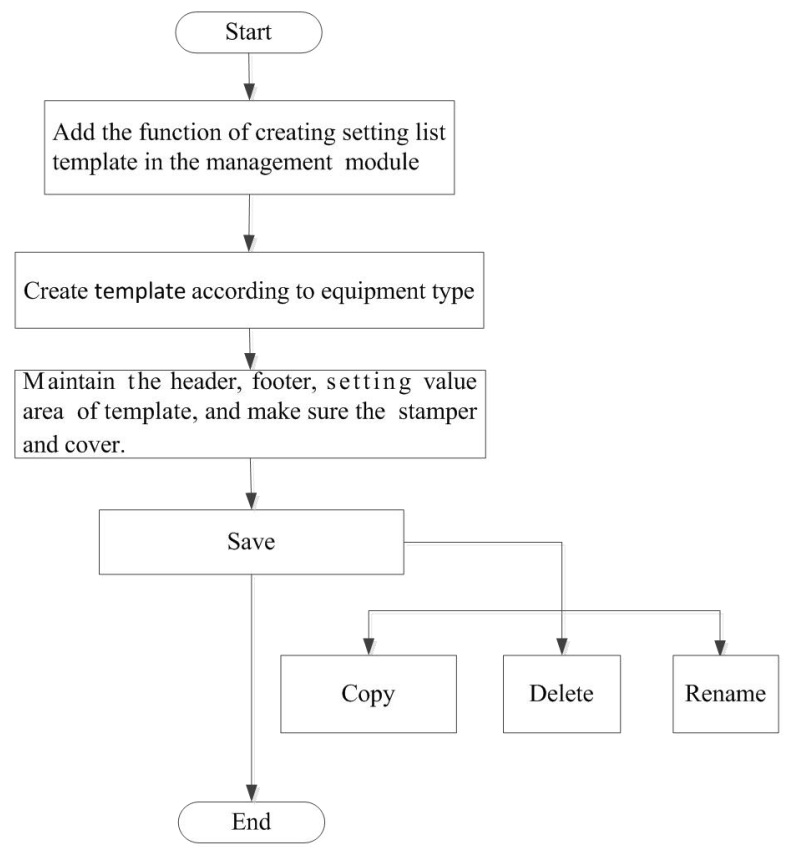

Fig. 5 Creation process on setting value template

Graining setting list template has strong flexibility, and has a good scalability and applicability. The specific setting list template was created by users according to the actual need, then to promote the application of setting calculation software.

\section{Example}

Based on six unified standard, the function of setting common libraries and graining setting value for protection device was applied to taizhou power grid in this article, in the case of line protection, implementation steps as follows:

(1) Using common libraries of protection device to create line protection device, and the new way was used to personalized setting value, the subscription way was adopted to establish common items from the common library;

(2) Using the technology of graining setting value to create the line setting template, and custom create the header, footer of template;

(3) Created setting list template was related to the protection model;

(4) Protection device was collocated on sample line, and set protection device, then generate setting list, the generated setting list was shown in figure 6:

Notice of relay protection setting value for state grid of Taizhou power supply company

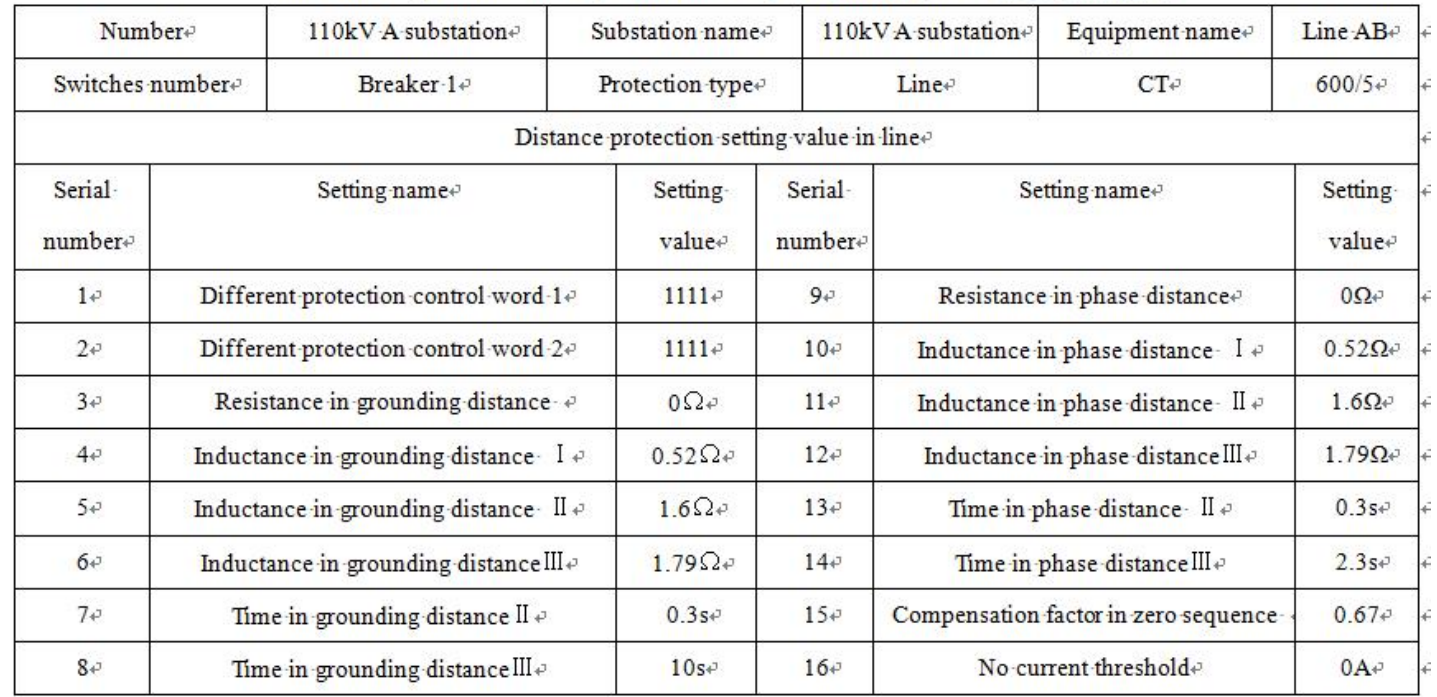

Fig. 6 Setting value list created by granular technology 
In figure 6, content such as title, header, content, setting value can change according to the needs of users, and different protection device can be used the same setting list template; setting value items will follow the change of the protection device, to solve the inflexible of template in current setting calculation software, the workload of establish template was greatly reduced.

\section{Conclusions}

The intelligent setting toolkit with setting common libraries and graining setting value based on six unified standard was introduced in relay protection setting software. This toolkit not only promoted the application of six unified standard, but also was suitable for the development requirements of the current power grid. When the protection device under six unified standard was used, common setting value items were autonomous added according to the actual condition, and create template of setting list. Then through configuring protection, setting protection by setting software, finally the generation of setting list was realized, and the workload in establishing protection device template was simplified, the production efficiency was greatly improved. Finally, through the practical application of taizhou power grid, confirmed the toolkit has a good practicability, expansibility and practicability.

\section{References}

[1] CUI Xiaodan, LI Bijun, LI Wei, et al. Operation mode optimization method of power grid with equipment monthly maintenance planning. Power System Protection and Control, 2016,44(09):102-107.

[2] LIU Qun. Research on the integration management of relay protection setting in Luoyang city and county power supply company. North China Electric Power University, 2014.

[3] ZHANG Wei. Development and application of relay protection setting calculation software. Agricultural University of Heibei, 2011.

[4] HAO Wenbin, HONG Xinglv. Key technology research on local net setting system of protective relaying setting for smart grid. Power System Protection and Control, 2011,39(2): 80-82.

[5] ZHOU Ding, DUAN Li, SHI Dongyuan, et al. Integrated system of protection setting calculation based on hybrid mode. Proceedings of the CSU-EPSA, 2007,19(3):109-112.

[6] WAGN Xiaofei, HU Jianzhi, WU Fangjie, et al. Improved adjacent matrix based sparse technology and its application in power system calculation. Power System Protection and Control, 2015,44(09):50-56.

[7] ZHAGN Jiankang, SU Xiaohua, XIA Yun. Discussion on protection configuration and setting calculation for $750 \mathrm{kV}$ transformer. Power System Protection and Control, 2015,(09):89-94.

[8] QIAN Hai, ZHAGN Zeng. Solutions to the problems encountered in the practical application of six-unified bus bar protection device. Northeast Electric Power Technology,2013, (7): 30-32.

[9] ZHAGN Guojing. The analysis of similarities and differences of line protection under the different design specification of four-unified and six-unified. Relay Protection Technology, 2013,(10): 9-12.

[10]Q_GDW 1161-2013, Standardization design specification of line protection and auxiliary.

[11]Q_GDW 1175-2013, Standardization design specification of transformers, high voltage shunt reactor and bus bar protection and auxiliary device.

[12]ZHANG Hailiang, SUN Wansheng. Design and development of general relay protection setting value management system. Electric power science and engineering, 2006,(3):72-74. 